International Journal of Engineering \& Technology, $7(2.21)(2018) 58-61$
International Journal of Engineering \& Technology
SPC
Website: www.sciencepubco.com/index.php/IJET
Research paper

\title{
Modelling of cloud based online access system for solar charge controller
}

\author{
S.S.P.M. Sharma B ${ }^{1 *}$, H. Ravishankar Kamath ${ }^{2}$, V. Siva Brahmaiah Rama ${ }^{3}$ \\ ${ }^{1}$ Assistant Professor, Department of Electrical, Mewar University, Rajasthan. \\ ${ }^{2}$ Professor, Department of ICE, Manipal University, Karnataka. \\ ${ }^{3}$ Assistant Professor, Department of Electrical, Mewar University, Rajasthan.
}

\begin{abstract}
In the current scenario, the considerable necessity of industry is to concatenate heterogeneous hardware components to the cloud based online access system for monitoring and identifying their behavior over a period. Every cloud computing is provided with a secured login credentials for limited access. The main objective of this paper is to record and transmit the readings of the instruments (Solar Charge Controller) to IoT which deals with establishing the suitable Web application on the cloud for Data logging and diagnostics. Using microcontroller information can be transmitted wirelessly with the help of Ethernet or Wi-Fi module. Some of the electrical devices can direct certain parameters to the microcontroller which are handled with the help of Ethernet or Wi-Fi module and connected to the cloud based system which may lessen the overall memory squandered. An attempt has been made to know the status of devices through PC/Laptop/Mobile/Tablet at any place while connected to cloud computing services.
\end{abstract}

\section{Introduction}

As there is an expeditious advancement in wired and remotely operated systems, smart devices incorporated with the web, i.e., Tablets, Cell Phones \& Laptops which were currently extensively deployed. Due to which the idea about Internet of Things (IoT) was instigated and acquired cogitation in last few years. Because of these, the subsequent technology for industrial products is IoT which predominantly concerns about cloud based systems and machine vision and analysis. The IoT can be disseminated from the embedded aspect in which Microcontroller is the soul of the proposed scheme, and the other is application aspect which is bestowed for cloud based web applications with secured login credentials. The transmission of information can be taken place in wired technique or remotely operated technique with the help of Microcontroller. The Ethernet or Wi-Fi module is coupled to Microcontroller with the help of Serial Communication (UART) which is connected to a cloud based system which may lessen the overall memory squandered. An attempt has been made to know the status of devices through PC/Laptop/Mobile/Tablet at any place while connected to cloud computing services. [1]

\section{Parameters consideration}

\section{Real time operating system}

The present reckoned system in this work is to provide concealment about the operating system which heeds about the basic functions and dispenses diversity in application services. In normal computers run on the common operating system but due to very less memory available in the embedded system it is involved with RTOS (Real Time Operating Systems). Microcontroller wiring based frame work is utilized in the proposed scheme without any built-in operating system not similar to Raspberry Pi. An open source Real time operating system is designed and utilized in the embedded system because of space allocated for memory and weight. It also extends a provision in providing memory managing \& handling the tasks and contrivances data types which are used to access the system. Programming of RTOS can be recorded in basic C-language and can be collated in other assembly languages. There is no limitation for task handling capability if there is a sufficient memory. [2]

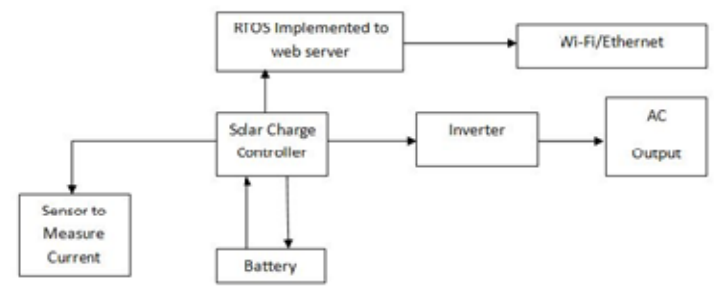

Figure 1: Proposed IoT application for solar charge controller using RTOS

\section{Structure of interaction using protocols}

A stow of communication protocols is used via the internet for interactions like TCP-IP which contribute an one to one communication concerning about in which manner unfinished data is the blockade and transmitted by way of the internet. The protocols TCP-IP is different in the case of IoT applications when compared with the normal application. For the embedded system the open source TCP-IP designed was lwIP which is currently arranged in the proposed scheme.

In the case of other than operating system environment, the transferring of TCP-IP is done in the following procedure. [3]

- $\quad$ Construct a file for the appearance of lwIP to compiler and architecture of the machine.

- Generate a configuration file for lwIP and select heterogeneous in protocols

- A network interface driver should be developed for communication 
- Design the software timer (definite functions are necessary to be operated at regular intervals).

- Connections of the hardware are to be verified by sharing the interactions with the various networks.

\section{Secure socket layer}

The major role of this layer is to yield a secured communication between client and server. The necessity of SSL is to provide.

- Data security throughout the internet (data format is done by HTTPS which ensures security, Packet size of 1408 and bandwidth of $2.4 \mathrm{Mbps}$ )
- Circumvent of deception of data which is utilized by the server to verify rectitude of client side which doesn't harm the server systems.

Every cloud based system is having its security credentials and the web services are authorized to permit data of client which are converted into a code by SSL which is anticipated to have validation from both client and server side. At the other end, Cloud based system anticipates only client side validation process. In this proposed scheme, Microcontroller Embedded SSL is used for security resolutions.

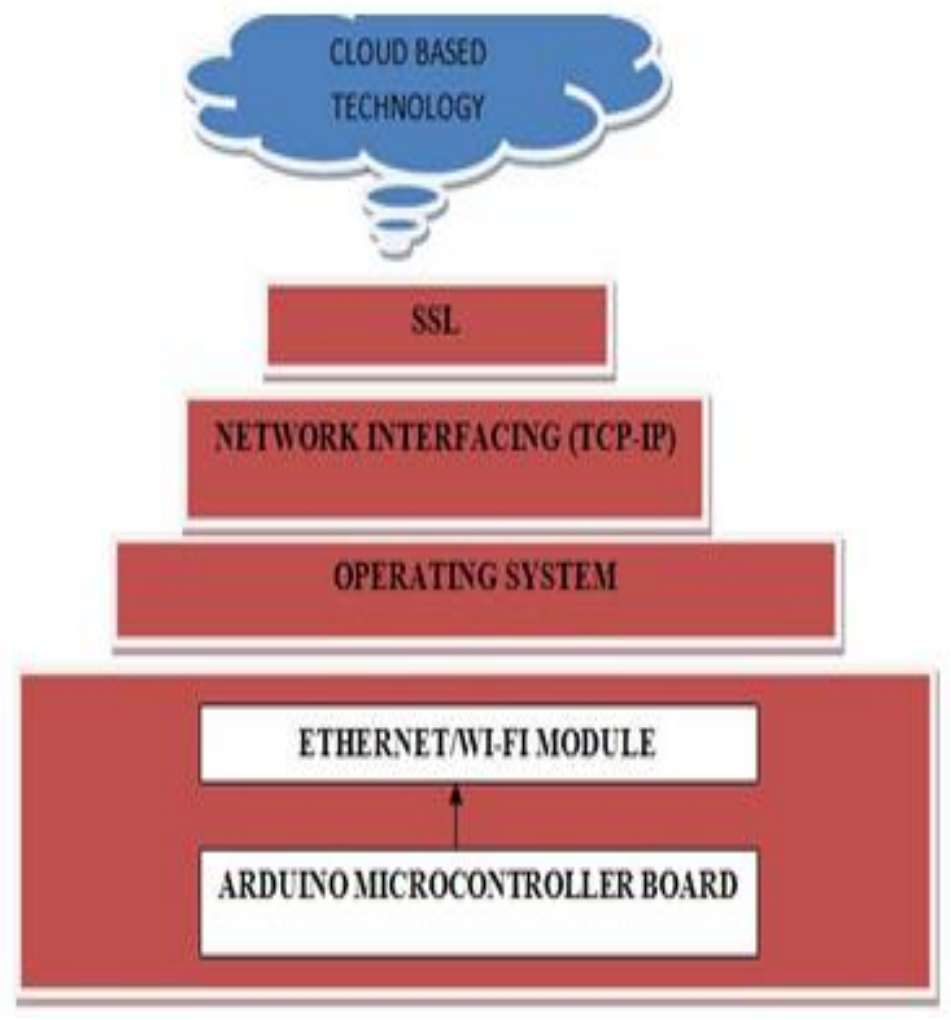

\section{Security of cloud based systems}

The IoT fulcrum vouchsafes a foremost connection string to end point devices. With the utility of the string, there can be an allocation of access given for generation of token which is PASSWORD for the cloud based security system. The cloud account will verify the token with the foremost connection string. The security verification process is straight forward in which connection of devices is strictly based memory limitation. This method doesn't provide any digital certificates. And the other procedure is devices are connected to cloud generates a digital certificate with the help of SSL provides an identity for devices. The diversity of information distributed by the devices is a version of a document, the action of time, locality. Any single device Id can earn mostly three certificates, i.e., client access, client cert, client key. [3]

\section{Solar charge controller}

Charge controllers are delineated to maximize output efficiency of a solar array. Charge controllers are utilized to oversee and manage solar array output voltages to batteries which are used to store the energy generated. Output voltage regulation is extremely predominant in the charging procedure of battery due to particular charging methodologies of enormous voltage and current levels which are required to extend the life and performance of the battery. Caliber charge controllers can be used in circumstances where the solar panel voltage is greater than the battery voltage. Charge controllers are used to having smart technologies such as micro controllers to attain maximum values of power at any given time. [4]

A modulated dc voltage is a prerequisite for battery charging. The dominant integrant in the solar battery charger is a solar panel, battery, converters \& controller. Due to the state of the weather and the flux of radiant energy per unit area from the sun can modify the supply voltage of solar panel. Impeccably a modulated voltage is essential for battery charging; a dc-dc converter is coupled betwixt battery and a solar panel. In the charge controller circuit the solar supply voltage is grabbed and convey to Microcontroller using serial communication (UART), and it is feasible to reserve solar supply voltage for a definite period of time in the cloud. The Pulse Width Modulation (PWM) is an efficient technique used to attain charging of the battery with persistent voltage is done by switching the power devices. Microcontroller is the soul of the charge controller, and its role is to sense the voltages of battery and solar panel and interacted across a Wi-Fi module with the help of Microcontroller. [4]

\section{Power supply}

The power from solar panels is required to power the current, voltage and other sensing circuits and also gate drivers' microcontroller and the wireless transmitter to achieve this buck converter is attached from the solar panel. Which is an Integrated Chip genre of converter should be adequate because the load which is connected to it is not dispositioned to draw a huge amount of currents? The essential voltages necessitate powering 
the Integrated Circuits of Charge controller $5 \mathrm{~V}$ to $3.3 \mathrm{~V}$ which is used for identification of right buck converter. [6]

LT3500 is a technology which is adept at delivering both DC voltage levels concurrently at $1 \mathrm{~A}$ rating of load current for individual outputs. An exemplar simulation of an LT3500 IC and brace circuitry, where a $24 \mathrm{~V}$ is treated as input (green in color) is lessened to a $5 \mathrm{~V}$ (blue) and a $3.3 \mathrm{~V}$ (red color) output voltages. The $1 \mathrm{~A}$ rating on both these rails will fortify constant power delivery to the charge controller's circuit and its ancillary. Attention is required for Integrated Circuit during the designing process. [7]

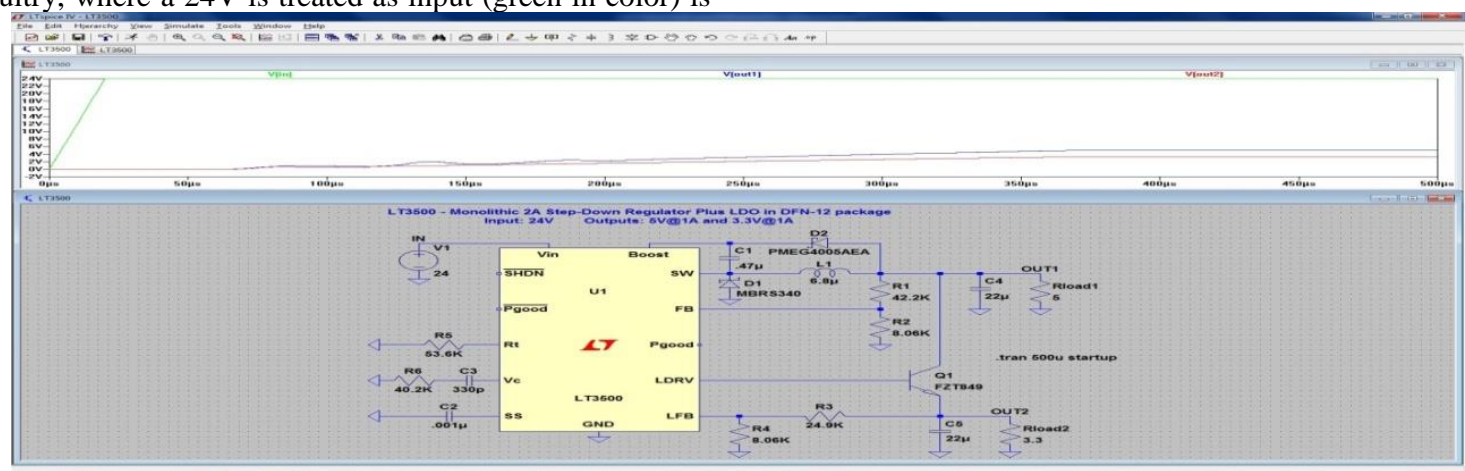

Figure 3: LT Spice simulation

3. Modeling of the system

\section{Voltage \& current sensor}

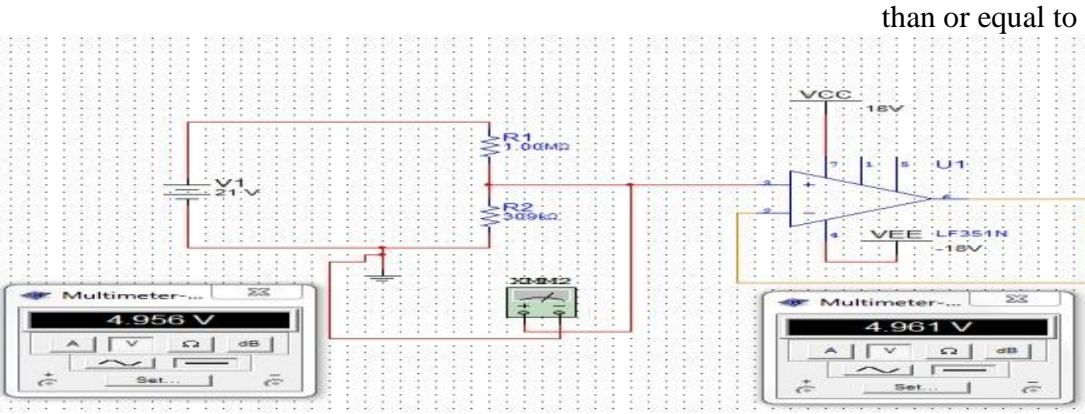

Figure 4: Voltage Sensor Circuit

To measure the voltage and current in the real time of the operating solar charge controller. A simulation of voltage sensor which is to be implemented is designed which deals with a supply of $21 \mathrm{~V}$ in worst case scenario and it is guaranteed to have less than or equal to $5 \mathrm{~V}$. [6]

\section{Temperature sensor}

The sensor is an integrated circuit which is utilized to measure the temperature in its surrounding premises. It computes and flaunts the values of temperature at regular intervals. The data acquired from the sensors is going to be displayed with the help of LCD. In this system temperature sensor perceive present temperature conditions and forwards to the Microcontroller.

\section{Wireless design}

The option available for generating a wireless data link is using a Wi-Fi module. The RNXV Wifly module communicates using the $802.11 \mathrm{~b} / \mathrm{g}$ protocol, and thus the device can be communicated with any computer or other device equipped with a wireless card using this same protocol. [7]

The wireless subsystem consists of two well-defined parts in which transmitting module which is located near the charge controller and the receiving module is located on a remote computer. At the end of the charge controller, the data collected by the sensor will be continuously fed through the microcontroller where it will be processed for transmission through Wi-Fi transceiver at the other end Wi-Fi module is connected via USB to the computer using Wi-Fi explorer board. From here data is processed and delivered to the users in an easy to read format.

This is to use two wireless transceivers to send or receive data. One device, the transmitter, will be a part of the charge controller. The other, the receiver, will be stationed at a computer within range of the wireless distance. This machine will take all the data received and deliver it to a user in a user-friendly manner using tables, graphs, etc. [7]

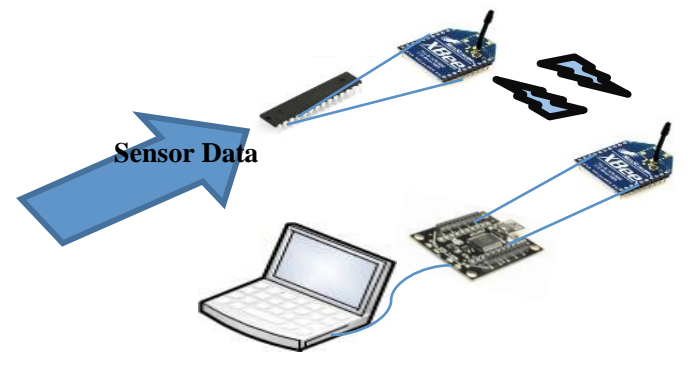

Figure 5: Wireless subsystem setup

\section{User interface}

Since Matlab is a widely used software package in both industry and academia, it will be the program of choice for developing a user interface. A graphical user interface (GUI) will be designed to showcase the sensor and system data.

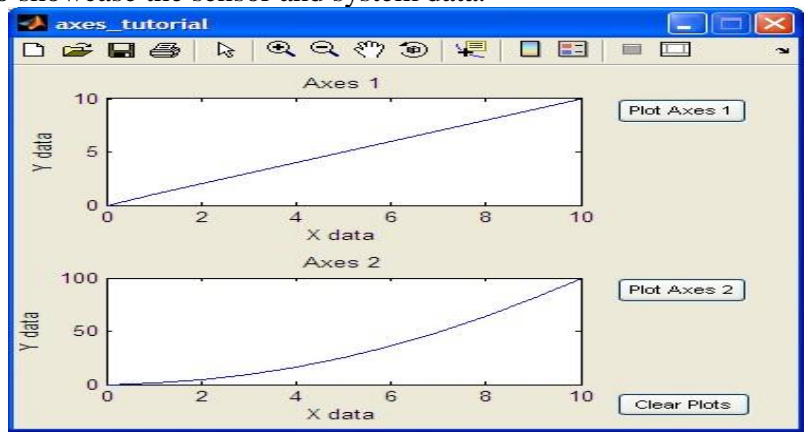

Figure 6: Matlab GUI design for data plotting 


\section{Experimental setup}

The entire setup of the system is consists of a microcontroller interfaced to sensors and converters and all electrical devices as shown. [1]

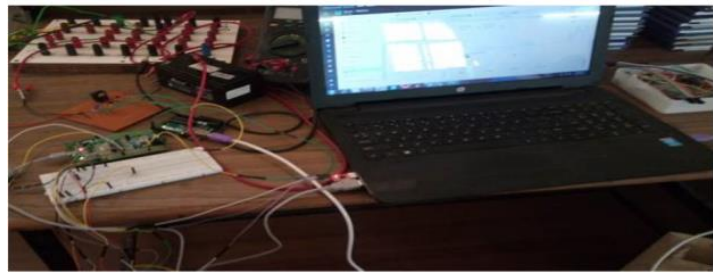

Figure 7: Setting up the entire system

\section{Merits}

- The system is delineated for memory limitations of the devices

- With analogize of other operating system boards utilizing micro controller boards are much cost effective.

- Majority of IoT are represented with an integration of Wi-Fi module with the microcontroller which may lead to substitution in case of any destruction happened to Wi-Fi module. But here using externally connected WiFi module can be replaced quickly in the event of damage occurs.

\section{Results}

Here we had estimated the data logging through cloud and readings are fetched to the microcontroller and further data is computed in MATLAB for plotting graph and updates the data on the cloud over a long duration.

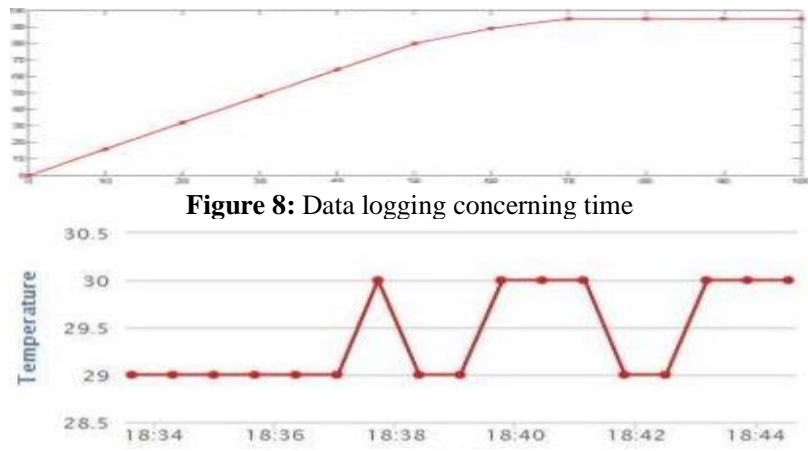

Figure 9: Registered temperature values

\section{Conclusion \& future scope}

The initial concept of modeling of this system has been modified make it more pertinent to industrial needs and practices. The most notable would be an inclusion of wireless potentiality. This adaptation is trusted to be crucial practicality to the modeling of the system. This is the feature which allows the users to not only monitor the system remotely but it can also be used to record and store the data, and this also felicitates with live monitoring conditions. [1]

The considerable benefit to both industries and research entities is creating live and historical data storage space. After certain period both could be taken advantage of the learning conditions related to atmospheric conditions affects the efficiency of systems. Meantime live monitoring can be intrinsically utilized for maintenance and intimate the users about any issues and unnecessary verifications. [1]

A formation of an association between micro controller and cloud was attained by deploying less memory usage. Electrical devices can interact and send their key parameters to micro controller which can transfer the data to the cloud system. The execution of the devices can be supervised by the data provided. A device working at a remote location of the world can be able to supervise from another place of the world. In future, sundry of devices can be able to communicate with them using the same technology. [2] Depending on the necessity, the wireless capability could be detached as well. The wireless capability would provide unique insight into the system status, solar efficiency, as well as allow for remote distress alerts. [3]

For example, in a large solar grid, it is not possible for all panels to have same irradiance levels at once, due to external factors. Because of this reason, a singular charge controller attempting to perform maximum power point tracking could not possibly allow the system to reach the maximum power point for each panel, therefore reducing the efficiency overall because it is addressing the system as a whole.[5]

\section{References}

[1] Stojmenovic I, "Machine-to-Machine Communications with InNetwork Data Aggregation, Processing, and Actuation for LargeScale Cyber Physical Systems", IEEE Internet of Things Journal, Vol.1, No.2, (2014), pp.122-128.

[2] Astley D, Dahlman E, Fodor G, Parkvall S \& Sachs J, "LTE Release 12 and Beyond", IEEE Commun. Mag., Vol.51, No.7, (2013), pp.154-160.

[3] Al-Fuqaha A, Guizani M, Mohammadi M, Aledhari M \& Ayyash, M, "Internet of things: A survey on enabling technologies, protocols, and applications", IEEE Communications Surveys \& Tutorials, Vol.17, No.4,(2015), pp.2347-2376.

[4] Lakeou S, Ososanya E, Latigo B \& Mahmoud W, "Renewable Power System for a Zero Energy Visitors' Center", 22nd European Photovoltaic Solar Energy Conference, 2007.

[5] Vilajosana I, Llosa J, Martinez B, Domingo-Prieto M, Angles A \& Vilajosana $X$, "Bootstrapping Smart Cities through a SelfSustainable Model Based on Big Data Flows", IEEE Commun. Mag., Vol.51, No.6, (2013), pp.128-134.

[6] Eclipse Paho Web page, eclipse.org/paho

[7] MQTT Website, http://mqtt.org

[8] Hohm DP \& Ropp ME, "Comparative Study of Maximum Power Point Tracking Algorithms", Progress in Photovoltaics: Research and Applications, Vol.11, (2002), pp.47-62.

[9] Lohner A, Meyer T and Nagel A, "A new panel-integrative inverter concept for grid-connected photovoltaic systems", Industrial Electronics, 1996. ISIE '96., Proceedings of the IEEE International Symposium on, Vol.2, (1996), pp.827-831.

[10] Woodward SW, "Maximum-Power-Point-Tracking Solar Battery Charger", Electronic Design, (1998), pp.114-118. 